V.2, N.1, 2018

DOI: $10.33947 / 2595-6264-v 2 n 1-3533$

\title{
CT²P: UM FRAMEWORK TRANSDISCIPLINAR ORIENTADO A PROCESSOS PARA APOIO À FASE DE DESIGN DO PRODUTO EM ENGENHARIA DE SISTEMAS
}

\section{CT'P : A TRANSDISCIPLINARY PROCESS ORIENTED FRAMEWORK TO SUPPORT THE PRODUCT DESIGN PHASE IN SYSTEMS ENGINEERING}

\author{
Renato Fernandes ${ }^{1}$
}

\begin{abstract}
RESUMO: O objetivo deste trabalho é apresentar e discutir as potencialidades de uma metodologia transdisciplinar de modelagem de processos, denominada Framework $\mathrm{CT}^{2} \mathrm{P}$, para apoiar os processos da fase de design de produto (desenvolvimento do produto e gestão da produção pela organização) no ciclo de vida de engenharia de sistemas. A metodologia consiste em criar um modelo conceitual de referência dos processos de ciclo de vida de engenharia de sistemas e transformá-lo em modelos especializados das áreas de Engenharia de Sistemas Baseada em Modelos, Gerenciamento de Projetos, Gestão de Processos de Negócios e Modelagem e Simulação. Os principais benefícios gerados pela abordagem são derivados de: A sistematização do desenvolvimento do modelo, englobando os processos de engenharia de sistemas e seu gerenciamento; $\mathrm{O}$ uso do Framework $\mathrm{CT}^{2} \mathrm{P}$ para implementação e análise de modelos, baseado no uso simultâneo de diversas disciplinas e suas respectivas metodologias e ferramentas; A avaliação conjunta dos modelos especializados criados para fornecer melhores soluções e melhorar o desenvolvimento do projeto. O Framework $\mathrm{CT}^{2} \mathrm{P}$ pode ser utilizado para a implementação de ambientes genéricos, feitos pelo conjunto de aplicações implementadas com as ferramentas especializadas utilizadas nas diversas disciplinas. Esses ambientes genéricos podem ser usados para realizar o gerenciamento do ciclo de vida de produtos por organizações que desenvolvem projetos de ciclo de vida curto, caracterizados por escopos de complexidade de baixo e médio nível, submetidos a severas restrições temporais e orçamentárias, como uma alternativa ao uso de sistemas proprietários complexos, caros e de difícil configuração, existentes no mercado.
\end{abstract}

PALAVRAS-CHAVE: Engenharia de Sistemas. Gerenciamento de Projetos. Gestão de Processos de Negócios. Simulação. Modelagem Transdisciplinar de Processos. Gerenciamento do Ciclo de Vida do Produto.

ABSTRACT: The objective of this work is to present and to discuss the potentialities of a transdisciplinary process modeling methodology, which has been named CT2P Framework, to support the product design phase processes (production and management) in the systems engineering lifecycle. The methodology consists in creating a conceptual reference model of the systems engineering lifecycle processes and to transform it into specialized models of the areas of (Model Based) Systems Engineering, Project Management, Business Process Management and Simulation Modeling. The main benefits generated by the approach are derived from: The systematization of the model development, encompassing both the systems engineering processes and their management; The use of the CT2P Framework for model implementation and analysis, based on the simultaneous use of diverse disciplines and their respective methodologies and tools; The joint assessment of the specialized models created to provide better solutions and to improve project development. The CT2P Framework can be used for the implementation of generic environments, made by the ensemble of applications implemented with the specialized tools used in the diverse disciplines. These generic environments can be used to perform product lifecycle management by organizations conducting short lifecycle project developments, characterized by low and mid-level complexity scopes submitted to severe time and budgetary constraints, as an alternative to the use of complex, expensive and difficult to configure proprietary systems existing on the market KEYWORDS: Systems Engineering. Project Management. Business Process Management. Simulation. Transdisciplinary Process Modeling. Product Lifecycle Management

\footnotetext{
Doutor em Engenharia de Sistemas Espaciais (INPE). Mestre em Engenharia Mecânica (UNITAU). Especialista em Engenharia da Computação (UFU). Graduado em Processamento de Dados (UFSC). Graduado em Computação (ITA). Professor da UNIVERITAS/UNG - Av. Anton Phillips, n01, Vila Hermínia - Guarulhos / SP- CEP: 07030-010 - renato.fernandez@prof.ung.br
} 
CT²P: UM FRAMEWORK TRANSDISCIPLINAR ORIENTADO A PROCESSOS PARAAPOIO À FASE DE DESIGN DO PRODUTO EM ENGENHARIA DE SISTEMAS Renato Fernandes

V.2, N.1, 2018

\section{INTRODUÇÃO}

Ciência e Tecnologia Transdisciplinares de Processos $\left(C T^{2} P\right)$ (Kienbaum et al. 2012 e Kienbaum, 2016) é um termo usado neste trabalho para designar uma visão holística, que consiste na integração de várias disciplinas que lidam com modelos de processos complexos de eventos discretos: Engenharia de Sistemas (Baseada em Modelos) (do inglês, (Model Based) Systems Engineering - SE) (SEBoK, 2018 e MBSE, 2018), Gerenciamento de Projetos (do inglês, Project Management - PM) (PMBOK, 2017), Gestão de Processos de Negócios (do inglês, Business Process Management - BPM) (Jeston e Nelis, 2017) e Modelagem e Simulação (do inglês, Simulation Modeling - SIM) (Pidd, 2004).

$\mathrm{O}$ objetivo geral da pesquisa em $\mathrm{CT}^{2} \mathrm{P}$ tem um escopo amplo e de natureza muito ambiciosa: o desenvolvimento de uma metodologia transdisciplinar madura e abrangente para modelagem de processos, e projetar e implementar seu ambiente de apoio integrado para a realização de estudos de uma grande categoria de problemas de processos complexos de eventos discretos, a fim de obter soluções melhores e integradas.

$\mathrm{O}$ domínio do problema coberto pela $\mathrm{CT}^{2} \mathrm{P}$ é essencialmente o mesmo abordado pela modelagem de processos de eventos discretos e procedimentos de simulação baseados em representações de modelagem, como Diagramas de Ciclo de Atividade (do inglês, Activity Cycle Diagrams - ACD) (Pidd, 2004), Diagramas de Atividades de Função (do inglês, Role Activity Diagrams - RAD) (Murdoch e McDermid, 2000) e Diagramas de Fluxo de Trabalho (do inglês, Workflows) (AALST, 2003).

A principal diferença e contribuição original entre o uso do Framework $\mathrm{CT}^{2} \mathrm{P}$ e aquelas disciplinas que tem o uso tradicional de seus componentes é a criação de um modelo transdisciplinar de processo e seu uso como base comum para o desenvolvimento de seus modelos individuais especializados de processos. Isso é feito através da realização de um procedimento sistemático de modelagem designado como Modelagem Conceitual ou de Referência e fazendo uso de uma notação gráfica denominada Diagramas de Modelagem Unificada do Ciclo de Vida (DMUCV), uma técnica inovadora de diagramação originada de uma fusão das representações gráficas opus citatus.. (Kienbaum et al. 2012 e Kienbaum, 2016).

\section{MÉTODOS}

Este trabalho apresenta a abordagem $\mathrm{CT}^{2} \mathrm{P}$ como uma aplicação totalmente integrada de suas metodologias e técnicas individuais, originadas de suas disciplinas autônomas (SE, PM, BPM, SIM) e de seus componentes, para a fase de design do ciclo de vida da Engenharia de Sistemas.

A limitação do escopo para a fase de design do ciclo de vida da Engenharia de Sistemas, a fim de explicar a abordagem $\mathrm{CT}^{2} \mathrm{P}$, foi feita apenas para fins de demonstração. Na verdade, ela pode ser aplicada a qualquer categoria de processos complexos de eventos discretos que possa ser tratada com a notação DMUCV e se destina a ser escalável e capaz de ser usada em um contexto mais amplo, cobrindo toda a gestão de processos de negócios da organização.

O restante deste trabalho está estruturado conforme segue. A seção 1 define alguns conceitos fundamentais usados na $\mathrm{CT}^{2} \mathrm{P}$. A seção 2 descreve a metodologia $\mathrm{e}$ sua aplicação. A seção 3 identifica o problema e define o escopo geral da pesquisa. A seção 4 apresenta a fase de modelagem conceitual e o meta-modelo de referência resultante. A seção 5 refere-se ao uso da metodologia para criar ambientes de Gerenciamento do Ciclo de Vida do Produto (do inglês, Product Lifecycle Management - PLM) básicos ou genéricos, feitos pelo conjunto de aplicações resultantes da implementação dos modelos especializados, utilizando as disciplinas autônomas e suas ferramentas de apoio. A seção 6 faz algumas considerações sobre o domínio de aplicabilidade da $\mathrm{CT}^{2} \mathrm{P}$ e os benefícios e limitações da metodologia. Finalmente, a Seção 7 apresenta as conclusões.

\section{FUNDAMENTOS DA METODOLOGIA}

Os conceitos mais fundamentais e diferenciados da $\mathrm{CT}^{2} \mathrm{P}$ são aqueles do modelo transdisciplinar de referência e sua notação específica, denominada DMUCV (Kienbaum et al. 2012 e Kienbaum, 2016). O modelo de referência em DMUCV descreve o processo de ciclo de vida do sistema em suas características estruturais e dinâmicas essenciais, com o especial propósito de servir como uma base comum para o desenvolvimento de modelos especializados adicionais a serem implementados em cada uma das disciplinas autônomas componentes da $\mathrm{CT}^{2} \mathrm{P}$. 
CT²P: UM FRAMEWORK TRANSDISCIPLINAR ORIENTADO A PROCESSOS PARAAPOIO À FASE DE DESIGN DO PRODUTO EM ENGENHARIA DE SISTEMAS Renato Fernandes

V.2, N.1, 2018

O formato da notação de modelagem assemelhase muito ao conjunto básico de ícones da Notação de Gestão de Processos de Negócios (do inglês, Business Process Management Notation - BPMN) (OMG, 2011) com algumas extensões, e pode-se questionar ainda a necessidade de "outro tipo de notação de modelagem de processo ou diagramas gráficos".

De fato, a BPMN é amplamente utilizada e suportada por grandes comunidades, tornando-se a notação "de fato" para criar modelos a serem convertidos em notações proprietárias específicas e implementados em uma variedade de ferramentas proprietárias oferecidas por diferentes fabricantes de sistemas.

O uso da notação DMUCV, no entanto, é apenas um primeiro passo (a fase de modelagem conceitual) de um processo de modelagem gradual e pode-se afirmar que algumas características semânticas da DMUCV são inexistentes em qualquer uma das tradicionais representações de modelos de processos gráficos ou textuais, como $A C D$, $R A D$, Workflows ou até mesmo BPMN. Essas características acabam sendo essenciais para o tipo de lógica e conhecimento representado no modelo de referência.

As características diferenciais da DMUCV começam com seu alto nível de abstração e minimalismo, compreendendo apenas 12 (doze) símbolos diferentes para expressar a estrutura completa e a dinâmica do ciclo de vida dos agentes como processos simultâneos, conforme mostrado na Figura 1.

A lógica do diagrama expressa uma multifuncionalidade: a decomposição do processo hierárquico, a variedade de agentes e mensagens e seus fluxos de controle de processos, os ciclos de vida ou papéis dos agentes e as interações entre eles. Os diagramas são criados na forma de módulos encapsulados, que podem ser apresentados em raias (faixas), descrevendo desde um processo muito simples (tarefa) até um muito complexo (macroprocesso).

\begin{tabular}{|c|c|c|}
\hline \multicolumn{2}{|c|}{ Designação } & \multirow[t]{2}{*}{ Descrição } \\
\hline DMUS / DMCU & DMUCV & \\
\hline $\mid \mapsto$ & & $\begin{array}{l}\text { Ponto de início do processo do ciclo de vida } \\
\text { de entidades }\end{array}$ \\
\hline & 0 & $\begin{array}{l}\text { Ponto de término do processo do ciclo de vida } \\
\text { de entidades }\end{array}$ \\
\hline & & Repositórios de entidades ou recursos \\
\hline N/A & & $\begin{array}{l}\text { Intersecção de entidades ou recursos entre dois processos } \\
\text { apresentados em raias ou frames separados }\end{array}$ \\
\hline \pm & & Macroprocesso \\
\hline & & Processo / Atividade Simples \\
\hline & & $\begin{array}{l}\text { Link mostrando of fluxo de controle de qualquer tipo ou número de } \\
\text { entidades, recursos, artefatos ou mensagens que estão sendo } \\
\text { transferidos }\end{array}$ \\
\hline N/A & & $\begin{array}{l}\text { Conexão entre fronteiras do fluxo de controle de quaisquer tipos de } \\
\text { entidades ou recursos. As fronteiras separam raias ou frames e os } \\
\text { simbolos descrevem respectivamente o envio e recebimento de } \\
\text { mecanismos. }\end{array}$ \\
\hline & & $\begin{array}{l}\text { Link mostrando o fluxo de controle de artefato ou mensagem } \\
\text { específica que está sendo transferida e que necessita ser } \\
\text { distinguida }\end{array}$ \\
\hline N/A & & $\begin{array}{l}\text { Conexão entre fronteiras do fluxo de controle de artefato ou } \\
\text { mensagem especifica sendo transferida, que necessitam ser } \\
\text { distinguidos. As fronteiras separam raias ou frames e os simbolos } \\
\text { descrevem respectivamente o envio e recebimento de mecanismos. }\end{array}$ \\
\hline$N / A$ & & Link mostrando o sincronismo entre dois processos \\
\hline N/A & & $\begin{array}{l}\text { Conector usado para direcionar o fluxo de controle entre os processo } \\
\text { para expressar roteamento em junçōes (ou exclusivo, separação e } \\
\text { uniäo), o qual também tem capacidade de processamento }\end{array}$ \\
\hline N/A & & $\begin{array}{l}\text { Bloco utilizado para roteamento, associados com os caminhos } \\
\text { percorridos por entidades, recursos, artefatos ou mensagens }\end{array}$ \\
\hline & N/A & $\begin{array}{l}\text { Fila de entidades/recursos esperando diante de uma atividade } \\
\text { prestes a se iniciar }\end{array}$ \\
\hline & N/A & $\begin{array}{l}\text { Fila de artefatos/mensagens esperando diante de uma } \\
\text { atividade prestes a se iniciar }\end{array}$ \\
\hline
\end{tabular}

Figura 1. Descrição dos símbolos da notação de modelagem DMUCV

Fonte: Fernandez, 2016.

Subjacente a todas essas características, algumas das quais ainda poderiam ser declaradas em relação à $B P M N$, há um recurso inigualável que diferencia a DMUCV de todos os outros tipos de diagramas de modelagem de processos existentes. Eles não são apenas diagramas sobre puras operações lógicas e fluxos de controle, eles são essencialmente atividades de transformação em tempo real executadas por entidades reais e mensagens (objetos) trocadas entre elas.

Os links correspondem a entidades reais que fluem ao longo de seu ciclo de vida (linhas sólidas) ou objetos (linhas pontilhadas) trocados entre as atividades, ambas usadas como entradas para outras atividades. Cada classe de entidade tem seu ciclo de vida específico de processo e interage para executar suas atividades comuns, que são interrompidas até que todas as entradas necessárias (entidades e/ou objetos) estejam disponíveis e as condições iniciais internas adicionais para sua 
CT²P: UM FRAMEWORK TRANSDISCIPLINAR ORIENTADO A PROCESSOS PARAAPOIO À FASE DE DESIGN DO PRODUTO EM ENGENHARIA DE SISTEMAS Renato Fernandes

V.2, N.1, 2018

execução, sejam atendidas.

Um tipo especial de condição é externo e é representado graficamente, denominado mecanismo de acionamento.

Os fluxos de controle são sempre associados a algum tipo de entidade, recurso, objeto ou mecanismo de acionamento, e eles reativam os processos em estado de espera em um determinado ponto no tempo e localização, fazendo com que ele progrida ao longo de seu ciclo de vida completo.

\section{O FRAMEWORK $\mathrm{CT}^{2} \mathrm{P}$}

$O$ estudo da $\mathrm{CT}^{2} \mathrm{P}$ é realizado utilizando o framework $\mathrm{CT}^{2} \mathrm{P}$ (Kienbaum et al. 2012 e Kienbaum, 2016), que consiste de três elementos, que constituem os pilares da metodologia: 1) Estrutura do Conhecimento, formada pelo modelo transdisciplinar do processo hierárquico criado (modelo conceitual ou de referência) e todas as informações adicionais sobre o sistema que está sendo estudado; 2) Método de Implementação, que é o método utilizado para a evolução dos modelos ao longo de seus respectivos ciclos de vida; 3) Ambiente de Apoio, que é constituído pelo conjunto integrado de ferramentas utilizadas e suas aplicações.

A Figura 2 apresenta a abordagem $\mathrm{CT}^{2} \mathrm{P}$ descrita como macroprocessos e suas respectivas atividades componentes. A primeira coluna mostra o procedimento do Método de Implementação ou Modelagem Unificada do Ciclo de Vida usado para criar os modelos especializados e evoluí-los em seus ciclos de vida.

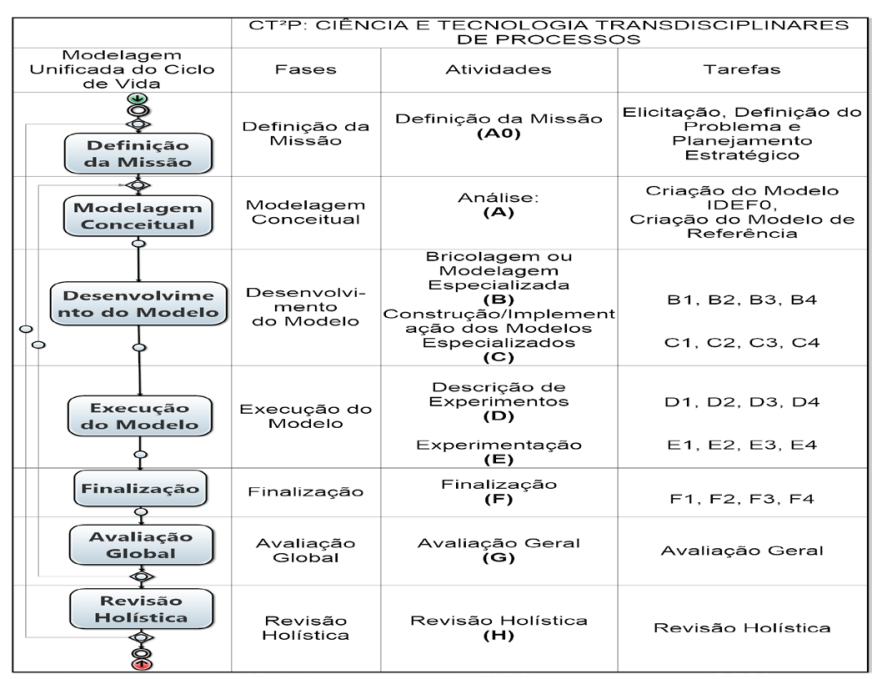

Figura 2. Metodologia para uso da Ciência e Tecnologia Transdisciplinares de Processos $\left(\mathrm{CT}^{2} \mathrm{P}\right)$ em Engenharia de Sistemas

Fonte: Fernandez, 2016
O Método de Implementação ou Modelagem Unificada do Processo de Ciclo de Vida do Sistema é criado pela agregação das diferentes visões de mundo usadas pelas disciplinas individuais que fazem parte da $C^{2} \mathrm{P}$ (SE, $P M$, $B P M, S I M)$. Essas disciplinas são aplicadas em conjunto, utilizando um modelo de referência como base comum para a uniformização da descrição do sistema, representativa de suas características estruturais e dinâmicas essenciais. O método de implementação compreende a transformação do modelo de referência em modelos especializados, sua implementação, execução, análise e avaliação, inicialmente como unidades, e posteriormente como agregados, gerando uma análise multidimensional completa do sistema, com base em uma visão holística que engloba todas as disciplinas individuais aplicadas.

A fase de Definição da Missão consiste na definição do sistema ou problema e na identificação dos objetivos do estudo, que é realizado em uma atividade dividida em três tarefas, denominadas Elicitação, Definição do Problema e Planejamento Estratégico, para fins de generalidade.

A segunda fase é a Fase de Modelagem Conceitual, para construir o meta-modelo de referência dos processos hierárquicos no formato DMUCV, descrevendo os processos operacionais, correspondentes às reais transformações ocorridas no sistema. Isso torna mais fácil para os diferentes tipos de usuários e modeladores, entenderem o comportamento do sistema e, posteriormente, traduzir essa lógica do modelo em seu tipo específico de notação, a fim de realizar as fases especializadas de desenvolvim ento e construção do modelo.

As fases restantes são aquelas mostradas na Figura 2 com suas respectivas denominações e definições mais detalhadas, dadas pela decomposição de seus respectivos macroprocessos em seus componentes, consistindo de atividades simples ou compostas, sendo as últimas denominadas tarefas.

Os quatro tipos numerados das Tarefas B, C, D, $\mathrm{E}$ e $\mathrm{F}$ são aqueles relacionados com as quatro disciplinas componentes da $C^{2}{ }^{2} P$ (SE, PM, BPM e SIM). Elas são tratadas separadamente durante as fases de Desenvolvimento do Modelo, Execução e Finalização (modelagem especializada, execução e avaliação), e seus resultados são integrados na Fase de Avaliação Global, para produzir uma avaliação completa do modelo multifacetado criado, utilizando o modelo transdisciplinar de referência. 


\section{V.2, N.1, 2018}

\section{DEFINIÇÃO DO PROBLEMA}

O SEBoK (Systems Engineering Body of Knowledge) (2018) [3] define que o ciclo de vida completo da Engenharia de Sistemas é composto por quatro (4) macroprocessos, denominados Fase de Design (Conceitual), Fase de Desenvolvimento, Operação e Descarte.

O domínio do problema de interesse neste trabalho é a fase de design do ciclo de vida da Engenharia de Sistemas (SE).

A Figura 3 mostra os principais processos da fase de design do ciclo de vida da SE: Definição de Conceitos e Definição de Sistemas e suas decomposições, os respectivos sequenciamentos e iterações (feedbacks) entre estes processos, obtidos do SEBoK, com a adição dos processos Análise de Viabilidade e Planejamento (Feasibility Analysis and Planning), a fim de adequá-lo aos padrões de gerenciamento de projetos e engenharia de sistemas espaciais.

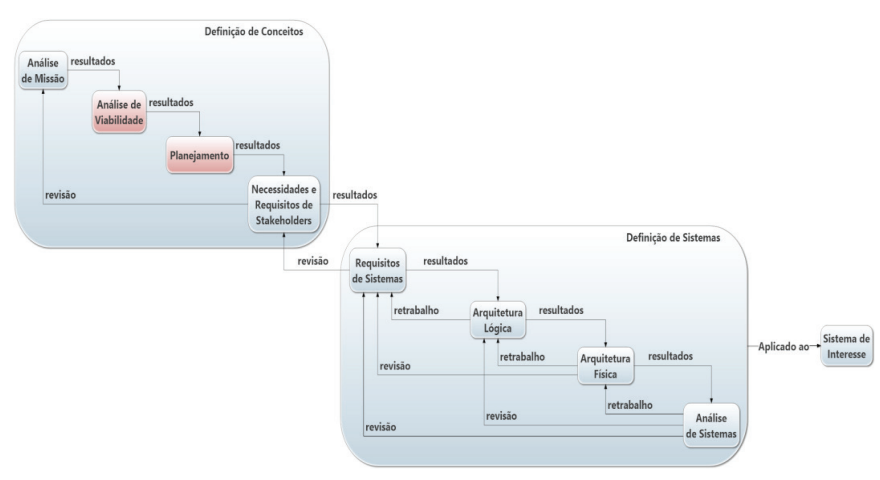

Figura 3. SEBoK (2018) Processos da fase de design da SE, modificada pelo autor.

Qualquer empresa que possua a Engenharia de Sistemas (Baseada em Modelos) (do inglês, (Model Based) Systems Engineering - MBSE) como o principal impulsionador de seus processos de produção, pode muito bem fazer uso dos conceitos e definições fornecidos no SEBoK (2018) para descrever a fase de design de seus produtos de acordo com essa estrutura.

Durante a execução do modelo, várias iterações e/ ou feedbacks sucessivos são executados, até que o resultado final seja alcançado, isto é, a definição e especificação completas do Sistema de Interesse (do inglês, System of Interest - Sol) sejam alcançadas.

\section{MODELO CONCEITUAL}

O procedimento da Modelagem Conceitual consiste na descrição formal do modelo através da realização de 02 (duas) atividades, a saber: a criação do Modelo IDEFO (do inglês Integrated Computer Aided Manufacturing DEFinition for Function Modelling) e a Criação do Modelo de Referência.

\subsection{CRIAÇÃO DO MODELO IDEFO}

IDEFO (FIPS, 1993) é um método voltado para modelar as decisões, ações e atividades de uma organização ou sistema. Modelos eficazes de IDEFO ajudam a organizar a análise de um sistema e a promover uma boa comunicação entre o analista e o cliente.

Os modelos IDEFO são frequentemente criados como uma das primeiras tarefas de esforço de desenvolvimento de um sistema.

Como uma ferramenta de comunicação, o IDEFO aprimora o envolvimento de especialistas no domínio e na tomada de decisões consensuais por meio de dispositivos gráficos simplificados.

Como uma ferramenta de análise, o IDEFO auxilia o modelador a identificar quais funções são executadas, 0 que é necessário para executar essas funções, o que o sistema atual faz certo e o que o sistema atual faz errado.

Cada atividade ou função no modelo IDEFO pode ser decomposta em várias camadas ou níveis. Os níveis subsequentes seguem as mesmas convenções dos anteriores, portanto, um modelo IDEFO completo é uma representação hierárquica dos processos de componentes, descritos por sua decomposição interna em atividades ou funções em qualquer número de níveis necessários, para alcançar o nível desejado de granularidade, para uma descrição completa e detaIhada do modelo.

$\mathrm{Na}$ criação do modelo IDEFO, faz-se a decomposição hierárquica dos macroprocessos descritivos do ciclo de vida sem a preocupação do sequenciamento dos processos.

O objetivo da criação do modelo IDEFO é identificar os próprios processos hierárquicos e seus parâmetros de interesse, tais como: entradas, saídas, recursos ou mecanismos utilizados em sua execução.

As entradas e saídas podem representar objetos (matérias-primas ou produtos acabados, respectivamente) $\mathrm{e}$ os controles são geralmente associados aos padrões e regras aos quais os processos são submetidos. 
$C^{2}$ P: UM FRAMEWORK TRANSDISCIPLINAR ORIENTADO A PROCESSOS PARAAPOIO À FASE DE DESIGN DO PRODUTO EM ENGENHARIA DE SISTEMAS Renato Fernandes

V.2, N.1, 2018

\subsection{CRIAÇÃO DO META-MODELO DE REFERÊNCIA}

A segunda tarefa da Fase de Modelagem Conceitual é a criação do Meta-Modelo de Referência, um modelo de processos baseado no modelo descritivo e na adição da sequência de execução ou fluxo de trabalho dos processos.

O fluxo de trabalho para execução de processos é adicionado neste estágio do ciclo de vida de desenvolvimento do modelo unificado, fazendo uso da notação DMUCV.

O modelo de referência genérico dos processos do ciclo de vida do sistema descreve o mapa completo de processos com seus respectivos sequenciamentos no tempo, os principais atores ou agentes e seus respectivos ciclos de vida dos processos, separados em raias (faixas) pelos quais esses atores ou agentes são responsáveis, os mecanismos de roteamento e o fluxo de controle para execução dos processos, os diversos tipos de retroalimentação, para realizar algum tipo de retrabaIho ou revisão de produtos e objetos que deveriam ter sido previamente realizados.

A notação DMUCV do meta-modelo de referência da fase de design da Engenharia de Sistemas é mostrado na Figura 4.

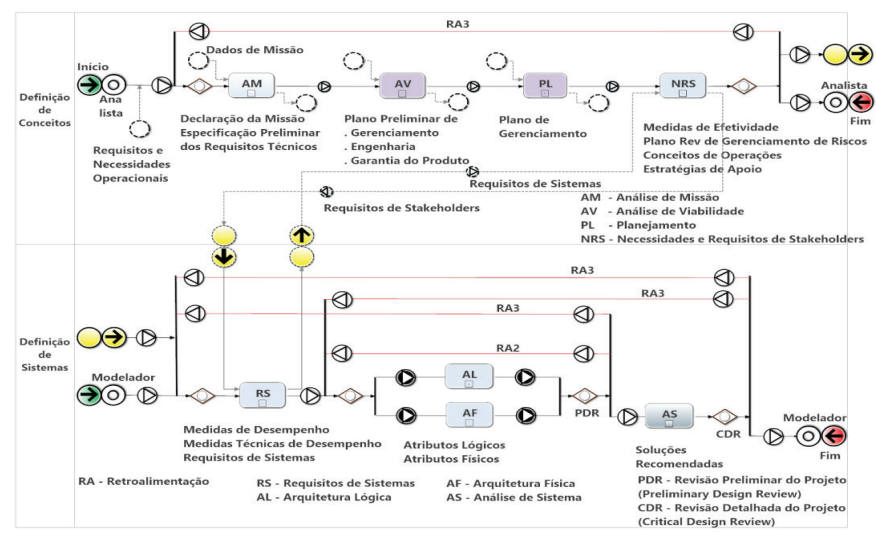

Figura 4. Fase de design da SE - definição de conceitos e definição de sistemas - em DMUCV

Fonte: Fernandez, 2016

O meta-modelo de referência completo compreende: processos de componentes exibidos hierarquicamente; seus principais atores ou agentes designados como entidades; seus respectivos ciclos de vida dos processos individuais com seus sequenciamentos no tempo; a descrição completa das entradas e saídas (objetos e mensagens consumidos ou gerados pelos processos); as regras de controle e normas utilizadas para as transformações; os recursos humanos e materiais ou mecanismos utilizados pelos processos; as interações e conexões exibidas dentro das raias (faixas) ou cruzando suas fronteiras.

Tudo isso é usado como um modelo de linha de base para a evolução para a próxima fase da representação do modelo, conhecida como Desenvolvimento de Modelos Especializados.

\subsection{CONSTRUÇÃO DO MODELO ESPECIALIZADO}

Os modelos especializados são criados gradativamente na forma de camadas, sendo a mais interna o modelo da Engenharia de Sistemas, representando as atividades de engenharia de sistemas previamente descritas no modelo genérico de referência, equivalentes aos processos principais ou atividades de transformação realizadas em tempo real, para o desenvolvimento de produtos ao longo de seu ciclo de vida, desde sua concepção inicial até sua etapa final.

O modelo de Engenharia de Sistemas expande o modelo de referência e representa a descrição mais completa da maneira como o sistema é desenvolvido, atuando como um sistema de controle de configuração.

Este tipo de modelo é um modelo descritivo, ou seja, não se destina a ser executado como um processo, destina-se a documentar a evolução do sistema com todas as informações importantes ao longo de todo o ciclo de vida de desenvolvimento do produto, semelhante a uma "receita" de como produzir o sistema, sob o ponto de vista da engenharia.

O modelo de Gerenciamento de Projetos estende o modelo da Engenharia de Sistemas com os processos de gerenciamento de projetos adicionais, ou seja, abrange as atividades necessárias para gerenciamento de projetos ao longo de todo o ciclo de vida do desenvolvimento do produto.

A criação do modelo de gerenciamento de projetos é considerada como a aplicação de técnicas de gerenciamento de projetos usando uma visão orientada a processos (PMBOK, 2017) apoiada por ferramentas de software.

O modelo de Gestão de Processos de Negócios, por sua vez, é construído no topo do modelo de gerenciamento de projetos e estende-se a quaisquer processos adicionais exigidos pela organização para o gerenciamento completo de seus processos de negócios, como 
CT²P: UM FRAMEWORK TRANSDISCIPLINAR ORIENTADO A PROCESSOS PARAAPOIO À FASE DE DESIGN DO PRODUTO EM ENGENHARIA DE SISTEMAS Renato Fernandes

V.2, N.1, 2018

por exemplo, aqueles relacionados à coordenação de um portfólio de projetos.

Esse procedimento gradual de modelagem e implementação prossegue até a camada mais externa a ser construída, o modelo de Simulação, que engloba todas as camadas internas, feitas pelo modelo de Engenharia de Sistemas com seus principais processos de desenvolvimento de produtos, o modelo de Gerenciamento de Projetos com os processos adicionais de gerenciamento de projetos e, finalmente, o modelo de Gestão de Processos de Negócios, com os processos complementares de gestão de negócios realizados pela organização durante o ciclo de vida completo de desenvolvimento de produto.

Qualquer uma das camadas individuais intermediárias pode ser tratada separadamente e usada para fins de análise, da maneira tradicional em que seus estudos são realizados.

A simulação como uma ferramenta também pode ser usada em associação com qualquer uma das camadas individuais em todo o procedimento de modelagem.

A denominação do modelo de Simulação, no entanto, é reservada para a quarta camada com os recursos adicionais necessários para conduzir um estudo de simulação completo do Sistema de Interesse.

\section{IMPLEMENTAÇÃO DE AMBIENTES GENÉRICOS DE APOIO AO PLM UTILIZANDO A CT ${ }^{2} P$}

PLM significa Gestão do Ciclo de Vida de Produtos, e seu valor está aumentando, especialmente para indústrias de manufatura, alta tecnologia e serviços. Na verdade, hoje o PLM é amplamente reconhecido como uma necessidade comercial para as empresas se tornarem mais inovadoras para enfrentar os desafios atuais, como personalização e rastreabilidade do produto, crescente concorrência, menor tempo de desenvolvimento e entrega de produtos, globalização, regulamentações mais rígidas e legislação.

A aplicação de ferramentas de software existentes, originadas de cada uma das áreas de estudo mencionadas, e a análise de seus resultados integrados na $\mathrm{CT}^{2} \mathrm{P}$, pode contribuir para a criação de um tipo de metodologia genérica de modelagem de PLM e construir um ambiente geral de apoio capaz de executar a modelagem e análise de processos de engenharia de sistemas e de gestão, em um contexto geral para melhorar significativamente a execução e o gerenciamento das atividades completas de ciclo de vida de gerenciamento de projetos.
Este ambiente genérico para gestão do ciclo de vida de produtos pode ser usado como alternativa ao uso de sistemas proprietários existentes no mercado que são complexos, caros e difíceis de serem configurados.

Seus potenciais usuários são principalmente as pequenas e médias empresas, que conduzem desenvolvimentos de projetos de sistemas tecnológicos altamente avançados, caracterizados por processos técnicos de baixo e médio nível de complexidade, ciclos de vida curtos de engenharia de sistemas e severas restrições financeiras.

\section{BENEFÍCIOS E LIMITAÇÕES DA CT²P}

$A C^{2} P$ apresenta uma alternativa interessante para melhorar a solução de problemas envolvendo processos complexos de negócios. A visão holística inovadora da $\mathrm{CT}^{2} \mathrm{P}$ aborda tanto a reestruturação do conteúdo do conhecimento (visão descritiva) quanto a criação de um procedimento geral de modelagem sistemática (visão unificada do processo), fazendo uso de modelos de referência para basear o uso individual ou combinado das diversas técnicas de modelagem para se criar modelos transdisciplinares multifacetados.

As disciplinas de Engenharia de Sistemas, Gerenciamento de Projetos, Gestão de Processos de Negócios e Modelagem e Simulação incorporadas na $\mathrm{CT}^{2} \mathrm{P}$, são disciplinas estabelecidas há muito tempo, com metodologias maduras e ferramentas de apoio, com uma grande comunidade de usuários, pois englobam uma ampla gama de métodos e técnicas usados para lidar com problemas de processos de eventos discretos em geral. Seus diferentes tipos de visões de mundo, técnicas e ferramentas de apoio são bem conhecidos por suas respectivas comunidades de usuários, embora haja uma falta de comunicação entre eles.

A visão holística da $\mathrm{CT}^{2} \mathrm{P}$ busca unificar os conceitos, métodos e ferramentas dessas diversas técnicas e aplicá-las de forma simples e consistente, integrando o trabalho de equipes multidisciplinares, alcançando os mesmos benefícios complementares de seu uso individual e, ao mesmo tempo, evitando quaisquer despesas gerais, inconsistências e duplicações relacionadas com a sua aplicação conjunta.

A metodologia proposta pode ser aplicada a uma grande classe de problemas que lida com sistemas complexos de processos de eventos discretos em geral. Essa abordagem holística poderia consolidar todo o conhecimento 
CT²P: UM FRAMEWORK TRANSDISCIPLINAR ORIENTADO A PROCESSOS PARAAPOIO À FASE DE DESIGN DO PRODUTO EM ENGENHARIA DE SISTEMAS Renato Fernandes

V.2, N.1, 2018

disperso em processos complexos sob um único guarda-chuva, capaz de cobrir todo o domínio do problema e abranger todo o ciclo de vida do modelo de processo.

Existe uma sobrecarga esperada nesta tentativa de aplicar uma abordagem transdisciplinar baseada em ferramentas autônomas existentes, representada pelo procedimento caro em relação à manutenção da consistência e compatibilidade do modelo em todo o ciclo de vida de desenvolvimento do modelo.

A sobrecarga resultante da aplicação de uma abordagem unificada desde o início do processo de modelagem é vista mais como uma antecipação de problemas futuros, que podem permanecer sem solução, se a maneira tradicional baseada na aplicação independente das técnicas e na coleta e interpretação de seus resultados para construir uma solução global, for aplicada.

Essa desvantagem pode ser reduzida por estudos futuros desse tipo, com o desenvolvimento de ambientes de apoio feitos por intermédio de uma montagem de ferramentas interoperáveis (Kienbaum, 2018) e o uso de procedimentos de verificação formal e de mecanismos de software para melhorar a consistência e compatibilidade do modelo do processo.

\section{CONCLUSÃO}

Os benefícios completos da abordagem transdisciplinar $\mathrm{CT}^{2} \mathrm{P}$ devem ser alcançados no longo prazo pelo projeto e implementação de ambientes híbridos de apoio ao PLM, capazes de lidar simultaneamente com todas as questões envolvidas nas áreas de estudos individuais.

Diferentes ambientes genéricos completos de apoio ao PLM deste tipo podem ser construídos para permitir a modelagem e análise de um processo de produção, como a aplicação de ferramentas básicas de Engenharia de Sistemas, Gerenciamento de Projetos, Gestão de Processos de Negócios e Modelagem e Simulação, em substituição a sistemas complexos de PLM (Kienbaum, 2018), que são caros e difíceis de personalizar. 
CT²P: UM FRAMEWORK TRANSDISCIPLINAR ORIENTADO A PROCESSOS PARAAPOIO À FASE DE DESIGN DO PRODUTO EM ENGENHARIA DE SISTEMAS Renato Fernandes

V.2, N.1, 2018

\section{BIBLIOGRAFIA}

AALST, Will M. P. van der; WESKE, Mathias; WIRTZ, Guido. "Advanced topics in workflow management: issues, requirements and solutions". Journal of Integrated Design \& Process Science. v. 7, Issue 3, August. 2003, p. 49-77. Disponível em: https://dl.acm.org/citation. cfm?id=1240310. Acesso em: 08 out. 2018.

Federal Information Processing Standards Publications (FIPS PUBS). Integration definition for function modeling (IDEFO). 1993. Disponível em: http://www.idef. com/wp-content/uploads/2016/02/ldef1x.pdf. Acesso em: 18 ago. 2018.

FERNANDEZ, Renato. "ENGESIS: um framework transdisciplinar orientado a processos para apoio à fase de design da engenharia concorrente em missões espaciais". Tese de Doutorado. INPE. São José dos Campos, 2016. Disponível em: http://mtc-m21b.sid.inpe.br/col/sid. inpe.br/mtc-m21b/2016/08.12.23.58/doc/publicacao.pdf. Acesso em: ?

JESTON, John; NELIS, Johan. Business process management: practical guidelines to successful implementations. 1 ed. Editora Elsevier, 2006.

KIENBAUM, Germano de Souza; SILVA, Luiz Alexandre da; LOUREIRO, Geilson; NETO, Álvaro Augusto; ROBINSON, Stewart. "A framework for process science and technology applied to concurrent engineering", In: ISPE International Conference on Concurrent Engineering, 2012, Trier, Germany. Proceedings. London: Springer-Verlag, 2012. v. 2, p. 1033-1044. ISBN 9781447144250. Disponível em: http://plutao.sid. inpe.br/col/dpi.inpe.br/plutao/2012/11.28.14.40.58/doc/ CE2012.pdf. Acesso em: 18 nov. 2018

KIENBAUM, Germano de Souza. "A Framework for process science and technology and its application to systems concurrent engineering". INPE. São José dos Campos, 2016. Disponível em: http://mtc-m21b.sid.inpe. br/col/sid.inpe. br/mtc-m21 b/2015/10.29.12.14/doc/publicacao.pdf. Acesso em: 18 nov. 2018.
KIENBAUM, Germano de Souza; FERNANDEZ, Renato; SILVA, Elaino Kelson Teixeira da; MARIA Rodrigo; COICEV, Marcelo; GARTENKRAUT, Eduardo; RODRIGUES, Marcio; NETO, Alvaro Augusto; FERREIRA, Mauricio. "A Transdisciplinary process oriented framework to support generic PLM implementation for use by small and medium enterprises", In: ISPE2016 International Conference on Transdisciplinary Engineering. IOS Press Ebooks, v. 4, p. 808-817. DOI 10.3233/9781-61499-703-0-808. Disponível em: http://ebooks.iospress.nl/volumearticle/45466. Acesso em: 11 nov. 2018

MODEL-Based Systems Engineering (MBSE). Disponível em: : <http://www.omgwiki.org/MBSE/doku. php>. Acesso em: 08 nov. 2018.

MURDOCH John; McDERMID, John A. "Modelling engineering design processes with role activity diagrams". Journal of Integrated Design \& Process Science, v. 4, Issue 2, April 2000, p. 45-65. Disponível em: https://dl.acm.org/citation.cfm?id=1240310. Acesso em: 08 out. 2018.

OMG-Object Management Group. Business process modeling and notation. Versão 2.0, 2011. Disponível em: https://www.omg.org/spec/BPMN/2.0/. Acesso em: 18 out. 2018.

PIDD, Michael. Computer Simulation in Management Science. 5. ed. Editora IE-WILEY, 2004.

PMBOK. Um guia do conhecimento em gerenciamento de projetos. Guia PMBOK. 6. ed. Newton Square, PA: Project Management Institute, 2017.

SEBoK. Guide to the Systems Engineering Body of Knowledge (SEBoK), version 1.8, 2017. 1.183 p. Disponível em: https://www.sebokwiki.org/wiki/Download_SEBoK PDF. Acesso em: 08 nov. 2018. 